\title{
Wall Estimation from Stereo Vision in Urban Street Canyons
}

\author{
Tobias Schwarze and Martin Lauer \\ Institute of Measurement and Control Systems, Karlsruhe Institute of Technology, Karlsruhe, Germany
}

Keywords: Environment Perception, Geometry Estimation, Robust Plane Fitting.

\begin{abstract}
Geometric context has been recognised as important high-level knowledge towards the goal of scene understanding. In this work we present two approaches to estimate the local geometric structure of urban street canyons captured from a head-mounted stereo camera. A dense disparity estimation is the only input for both approaches. First, we show how the left and right building facade can be obtained by planar segmentation based on random sampling. In a second approach we transform the disparity into an elevation map from which we extract the main building orientation. We evaluate both approaches on a set of challenging inner city scenes and demonstrate how visual odometry can be incorporated to keep track of the estimated geometry.
\end{abstract}

\section{INTRODUCTION}

Robotic systems aiming at autonomously navigating public spaces need to be able to understand their surrounding environment. Many approaches towards visual scene understanding have been made, covering different aspects such as object detection, semantic labeling or scene recognition. Also the extraction of geometric knowledge has been recognised as important high-level cue to support scene interpretation from a more holistic viewpoint. Recent work for instance demonstrates the applicability in top-down reasoning (Geiger et al., 2011a; Cornelis et al., 2008).

Extracting geometric knowledge appears as hard task especially in populated outdoor scenarios, because it requires to tell big amounts of unstructured clutter apart from the basic elements that make up the geometry. This problem can be approached from many sides, clearly depending on the input data. In recent years the extraction of geometric knowledge from single images has attracted a lot of attention and has been approached in different ways, e.g. as recognition problem (Hoiem et al., 2007), as joint optimization problem (Barinova et al., 2010), or by geometric reasoning e.g. on line segments (Lee et al., 2009). Other than the extensive work in this field, here we investigate the problem based on range data acquired from a stereo camera setup as only input, which is in principle replaceable by any range sensor like LIDAR systems or TOF cameras. We aim at extracting a local geometric description from urban street scenarios with building facades to the left and right ("street canyon"). Rather than trying to explain the environment as accurately as possible, our focus is a simplified and thus very compact representation that highlights the coarse scene geometry and provides a starting point for subsequent reasoning steps. To this end our goal is a representation based on geometric planes, in the given street canyon scenario one plane for each building facade, which are vertical aligned to the groundplane.

Such representation can basically be found in two ways. The 3D input data can be segmented by growing regions using similarity and local consistency criteria between adjacent data points that lead to planar surface patches, or surfaces can be expressed as parametric models and directly fitted into the data. Either way has attracted much attention. Studies on range image segmentation have been conducted, but usually evaluating range data that differs strongly from outdoor range data obtained by a stereo camera in terms of size of planar patches and level of accuracy (Hoover et al., 1996). Variants of region growing can be found in e.g. (Gutmann et al., 2008; Poppinga et al., 2008).

The combination of short-baseline stereo, large distances in urban scenarios and difficult light conditions due to a free moving and unconstrained platform poses challenging conditions. Additionally we can not assume free view on the walls since especially traffic participants and static infrastructure often occlude large parts of the images - a key requirement is hence robustness of the fitting methods.

Region growing alone does not guarantee to result 

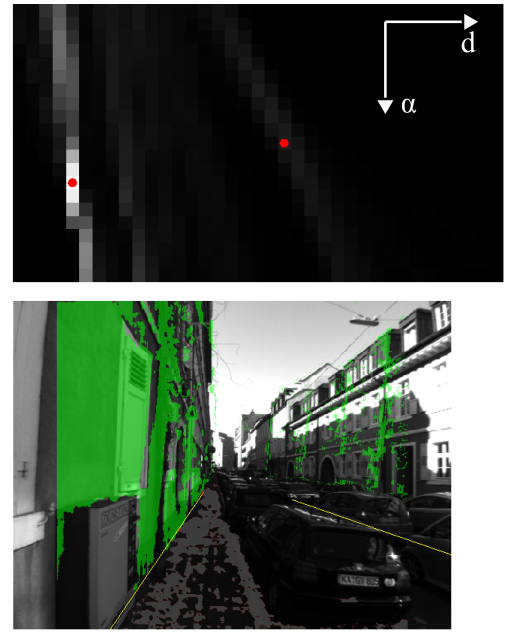

(a) $X Y Z$-points, fixed distance threshold
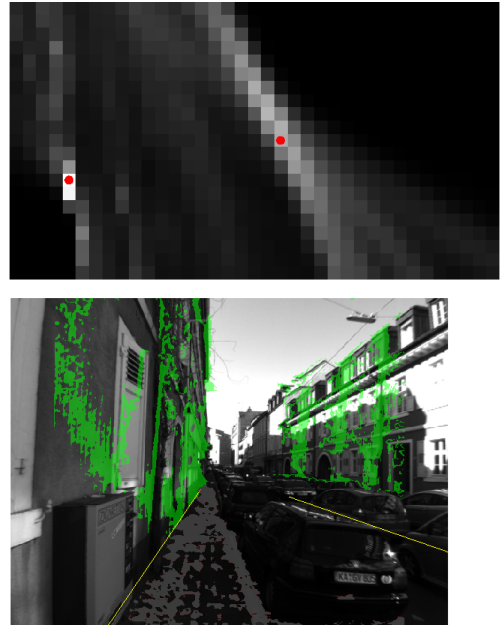

(b) XYZ-points, distance threshold scaled by covariance
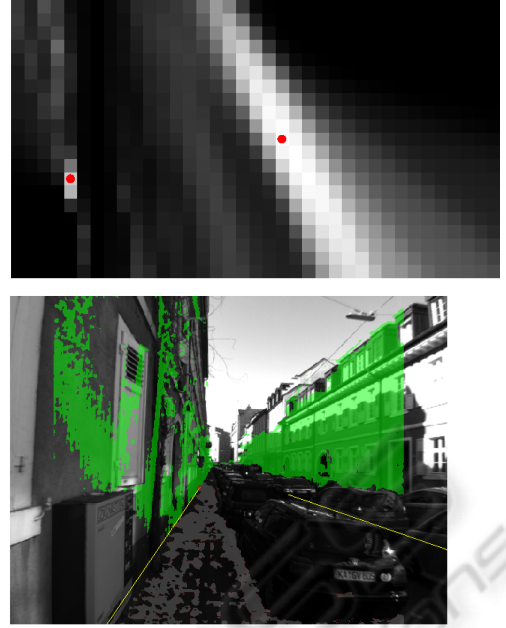

(c) $u v \delta$-points, fixed distance threshold

Figure 1: Plane estimation based on point-to-plane distance thresholds using (a) a fixed distance in $X Y Z$ coordinates, (b) the Mahalanobis distance in $X Y Z$ coordinates and (c) a fixed distance in $u v \delta$ coordinates. The top row visualizes with gray values the number of support points for a vertical plane swept through the $X Y Z$ - resp. $u v \delta$-points in distance and angle steps of $0.5 \mathrm{~m}$ resp. $1^{\circ}$. The true plane parameters are marked in red. The bottom row shows support points for these plane parameters as overlay.

in connected surfaces when occlusions visually split the data, a subsequent merging step would be necessary. This does not occur when parametric models are fitted directly. Most popular methods here are random sampling and 3D Hough transformations (Iocchi et al., 2000).

A large body of literature focuses specifically on the task of groundplane estimation, in case of vision systems planes have been extracted using v-disparity representations (Labayrade et al., 2002) and robust fitting methods (Se and Brady, 2002), often assuming fixed sensor orientation (Chumerin and Van Hulle, 2008).

We start with estimating the groundplane using random sampling. Based on the groundplane parameters we constrain the search space to fit two planes to the left and right building facade. In Section 2.2 and 2.3 we present two robust methods to fulfil this task. In Section 3 we evaluate both methods using a dataset of inner city scenes and show how visual odometry data can be integrated to keep track of the estimated geometry.

\section{PLANE FITTING}

Estimating planar structures from an egocentric viewpoint in urban environments has to deal with a huge amount of occlusions. Especially the groundplane is often only visible in a very small part of the image since buildings, cars or even pedestrians normally occlude free view onto the ground. Hence, robustness of the methods is a key requirement. Therefore, we developed an approach based on the RANSAC scheme (Fischler and Bolles, 1981), which is known to produces convincing results on model fitting problems even with way more than $50 \%$ outliers. In a scenario with fairly free view and cameras pointing towards the horizon with little tilt a good heuristic is to constrain the search space to the lower half of the camera image space to find an initial estimate of the groundplane.

A plane described through the equation

$$
a X+b Y+c Z+d=0
$$

can be estimated using the RANSAC scheme by repeatedly selecting 3 random points and evaluating the support of the plane fitting these points. A fit is evaluated by counting the $3 \mathrm{D}$ points with point-to-plane distance less than a certain threshold. In our case, we had to extend the RANSAC scheme by an adaptive threshold to cope with the varying inaccuracy of 3D points determined from a stereo camera. To account for the uncertainty, the covariance matrices of the $X Y Z$ points can be incorporated into the distance threshold. In case of reconstructing from stereo vision one obtains the $3 \mathrm{D}$ coordinates $(X, Y, Z)^{1}$ through:

\footnotetext{
${ }^{1}$ Our Z-axis equals the optical axis of the camera, Xaxis pointing right and $\mathrm{Y}$-axis towards the ground. Compare Figure 2
} 


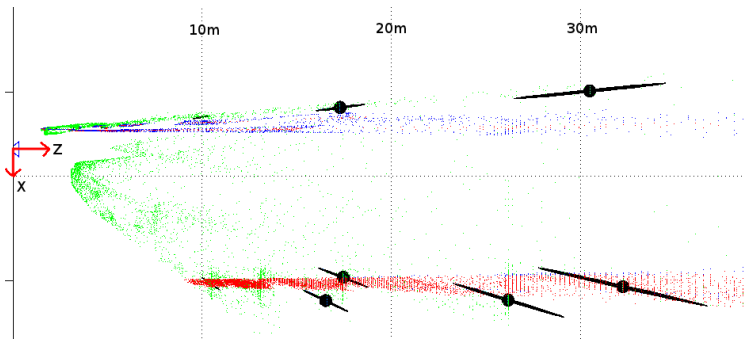

Figure 2: Stereo covariances.

$$
F(u, v, \delta)=\left[\begin{array}{c}
X \\
Y \\
Z
\end{array}\right]=\left[\begin{array}{c}
\frac{B\left(u-c_{x}\right)}{\delta} \\
\frac{B\left(v-c_{y}\right)}{\delta} \\
\frac{B f}{\delta}
\end{array}\right]
$$

Where $B$ is the baseline, $f$ the focal length, $\delta$ the disparity measurement at image point $(u, v)$, and $\left(c_{x}, c_{y}\right)$ the principal point. The covariance matrix $C$ can be calculated by (also found in (Murray and Little, 2004)) $C=J \cdot M \cdot J^{T}$ with $J$ the Jacobian of $F$

$$
J=\left[\begin{array}{lll}
\frac{d F_{X}}{d u} & \frac{d F_{X}}{d v} & \frac{d F_{X}}{d \delta} \\
\frac{d F_{Y}}{d u} & \frac{d F_{Y}}{d v} & \frac{d F_{Y}}{d \delta} \\
\frac{d F_{Z}}{d u} & \frac{d F_{Z}}{d v} & \frac{d F_{Z}}{d \delta}
\end{array}\right]=\left[\begin{array}{ccc}
\frac{B}{\delta} & 0 & \frac{-B\left(u-c_{x}\right)}{\delta^{2}} \\
0 & \frac{B}{\delta} & \frac{-B\left(v-c_{y}\right)}{\delta^{2}} \\
0 & 0 & \frac{-B f}{\delta^{2}}
\end{array}\right]
$$

Assuming a measurement standard deviation of $1 \mathrm{px}$ for the $u$ and $v$ coordinates and a disparity matching error of $0.05 \mathrm{px}$ we obtain as measurement matrix $M=\operatorname{diag}(1,1,0.05)$. A world point on the optical axis of the camera in $15 \mathrm{~m}$ distance is subject to a standard deviation of $\sim 1 \mathrm{~m}$ (focal length $400 \mathrm{px}$, baseline $12 \mathrm{~cm}$ ). While the $Z$ uncertainty of reconstructed points grows quadratically with increasing distance, the uncertainty of reconstructed $X$ and $Y$ components remains reasonable small (see Figure 2).

With the covariance matrices we can determine the point to plane Mahalabobis distance and use it instead of a fixed distance threshold to count plane support points. This way the plane margin grows with increasing camera distance according to the uncertainty of the reconstruction. Calculating the point to plane Mahalanobis distance essentially means transforming the covariance uncertainty ellipses into spheres. A way to do so is shown in (Schindler and Bischof, 2003).

However, calculating the covariance matrix for every $X Y Z$-point is computationally expensive. We can avoid this by fitting planes in the $u v \delta$-space and transforming the plane parameters into $X Y Z$-space afterwards.

Fitting planes in $u v \delta$-space can be done in the same way as described above for the $X Y Z$-space. We obtain the plane model satisfying the equation

$$
\alpha u+\beta v+\gamma-\delta=0
$$

Expressing $u, v, \delta$ through equations (1) yields the $u v \delta$ to $X Y Z$-plane transformation

$$
a=\alpha ; \quad b=\beta ; \quad c=\frac{\alpha c_{x}+\beta c_{y}+\gamma}{f} ; \quad d=-B
$$

and vice-versa

$$
\alpha=\frac{a B}{d} ; \quad \beta=\frac{b B}{d} ; \quad \gamma=\frac{B\left(c f-a c_{x}-b c_{y}\right)}{d}
$$

In the following section we demonstrate the importance of considering the reconstruction uncertainty when setting the plane distance threshold.

\subsection{Sweep Planes}

For the purpose of estimating facades of houses we construct a plane vertical to the groundplane and sweep it through the $X Y Z$ - respectively $u v \delta$-points. For most urban scenarios it is a valid assumption that man-made structures and even many weak and cluttered structures like forest edges or fences are strongly aligned vertical to the floor. Knowledge of the groundplane parameters $\left\{\vec{n}_{g p}, d_{g p}\right\}$ with groundplane normal $\overrightarrow{n_{g}}$ and distance $d_{g p}$ from the camera origin allows us to construct arbitrary planes perpendicular to the ground.

We construct a sweep plane vector perpendicular to $\overrightarrow{n_{g p}}$ and the $Z$-axis (compare Figure 2) by

$$
\vec{n}_{\text {sweep }}(\alpha)=R_{\vec{n}_{g p}}(\alpha)\left(\vec{n}_{g p} \times\left[\begin{array}{l}
0 \\
0 \\
1
\end{array}\right]\right) \text {, }
$$

where rotation matrix $R_{\vec{n}_{g p}}(\alpha)$ rotates $\alpha$ degrees around the axis given by $\vec{n}_{g p}$.

We sweep the plane $\left\{\vec{n}_{\text {sweep }}(\alpha), d_{\text {sweep }}\right\}$ through the $X Y Z$ respectively $u v \delta$-points by uniformly sampling $\alpha$ and $d_{\text {sweep }}$ and store the number of support points for every sample plane in the parameter space $\left(\alpha, d_{\text {sweep }}\right)$. The result for a sampling between $-10^{\circ}<\alpha<10^{\circ}$ and $-3 \mathrm{~m}<d_{\text {sweep }}<15 \mathrm{~m}$ with a stepsize of $1^{\circ}$ resp. $0.5 \mathrm{~m}$ is shown in Figure 1. Peaks in the parameter space correspond to good plane support.

A fixed threshold in $X Y Z$-space (Figure 1(a)) overrates planes close to the camera (left facade), while planes further away are underrated (right wall). The Mahalanobis threshold (Figure 1(b)) can compensate for this. Fitting planes in $u v \delta$-space leads to the same result (Figure 1(c)), without the computational expense.

The method can be used to extract planar surfaces from the points, regardless whether input data is present in $X Y Z$ - or $u v \delta$-space. It is easy to incorporate prior scene knowlegde like geometric cues to select 
planes and suppress non-maxima. Parallel planes are mapped to the same rows in parameter space, while a minimal distance between selected planes can be enforced by the column gap.

In practice, when knowledge about the rough orientation of the scene is unavailable, the computational cost of building the parameter space is very high. In our strongly constrained example scene with $\pm 10^{\circ}$ heading angle and $18 \mathrm{~m}$ distance already 777 planes had to be evaluated. The required size and subsampling of parameter space is hard to anticipate and would have to be chosen much bigger, which makes the approach less attractive in this simple form.

Due to the fact that the plane sweeping is not a data-driven approach, many planes are evaluated that are far off the real plane parameters. Simplifying the data does not affect the number of evaluated planes. In its data-driven form this algorithm resembles the Hough transform (Hough, 1962), which has also been studied and extended for model-fitting problems in 3D data, e.g. (Borrmann et al., 2011).

In the following sections we present two datadriven approaches for wall estimation. First, we show how RANSAC can be used to achieve a planar scene segmentation, from which we extract the street geometry. In Section 2.3 we use a 2D Hough transform to find the left and right facade.

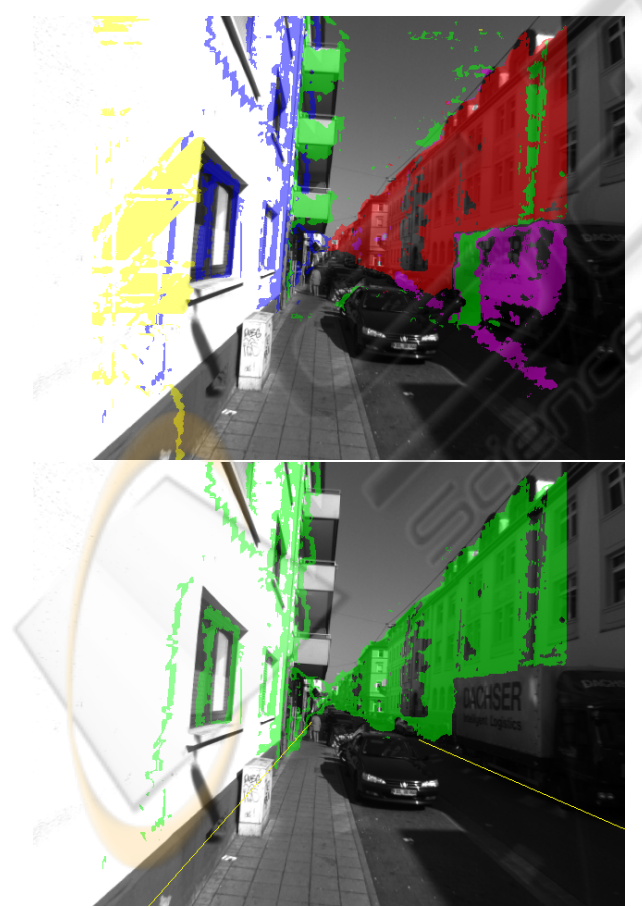

Figure 3: RANSAC based planar segmentation. The two most parallel planes (bottom) are selected from five hypotheses (top).

\subsection{Planar RANSAC Segmentation}

RANSAC based plane fitting can obviously not only be used to fit the groundplane but any other planar structure in the scene. Assuming the groundplane parameters are known, we first remove the groundplane points from $u v \delta$-space. In the remaining points we iteratively estimate the best plane using RANSAC. Since we are interested in vertical structures, we can reject plane hypotheses that intersect the groundplane at smaller angles than $70^{\circ}$ by comparing their normal vectors. After every iteration we remove the plane support points from $u v \delta$-space. This way we generate five unique plane hypotheses, out of which we select the two most parallel planes with distance $d=\left|\vec{n}_{1} d_{1}+\vec{n}_{2} d_{2}\right|>5 m$ by pairwise comparison to obtain the left and right building facade. Figure 3 shows an example scene with five plane hypotheses (top) out of which the red and blue one are selected since they are the most parallel and also exceed the minimal distance threshold.

The top row in Figure 4 presents the output for some challenging scenarios, some of which feature considerable occlusions. In every iteration we evaluate 50 planes with a valid groundplane intersection angle, 5 iterations hence summing to 250 evaluated plane hypotheses.

\subsection{Elevation Maps}

The strong vertical alignment of man-made environments can be exploited by transforming the 3D point data into an elevation map. We do this by discretizing the groundplane into small cells $($ e.g. $10 \times 10 \mathrm{~cm})$ and projecting the $3 \mathrm{D}$ points along the groundplane normal onto this grid. The number of 3D points projecting onto a cell provides a hint about the elevation over ground for the cell. Grid cells underneath high vertical structures will count more points than grid cells underneath free-space. Now, the grid can be analysed using 2D image processing tools. In case of a street scenario with expected walls left and right we apply a Hough transform to discover two long connected building facades. Because of geometric plausibility we again enforce a minimal distance of $5 \mathrm{~m}$ between walls when selecting the Hough peaks. Two examples along with their elevation map are shown in Figure 5.

The approach also works with weak structures like forest edges (bottom image), though overhanging trees are obviously causing a deviation from the real forest bottom here and the assumed street model with two walls does not hold in this view. The approach will benefit strongly from integrating elevation maps over multiple frames, which is topic of future work. 

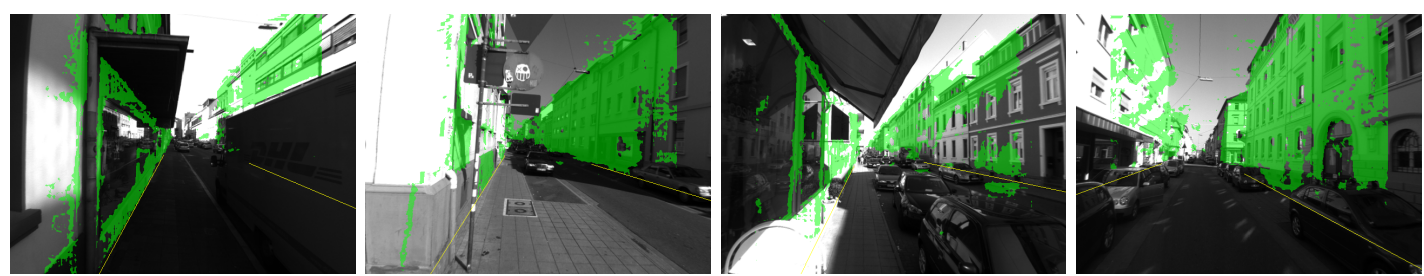

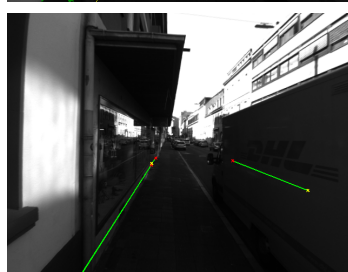

(a)
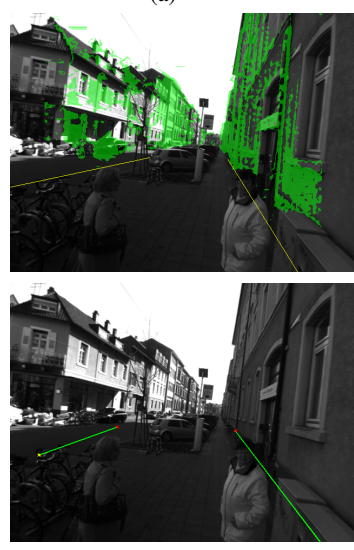

(e)

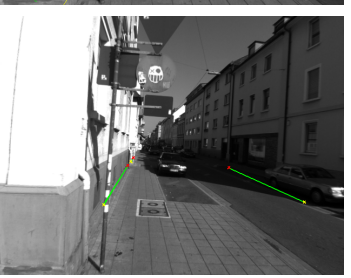

(b)
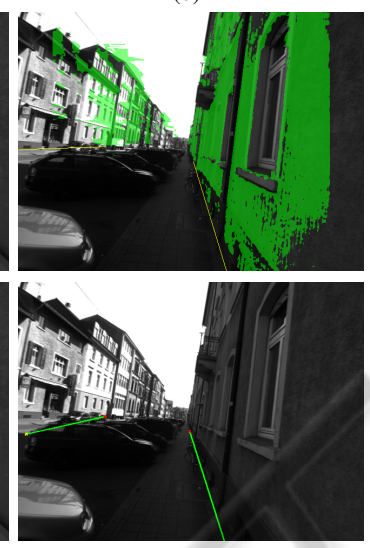

(f)

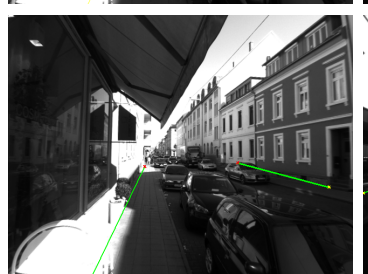

(c)

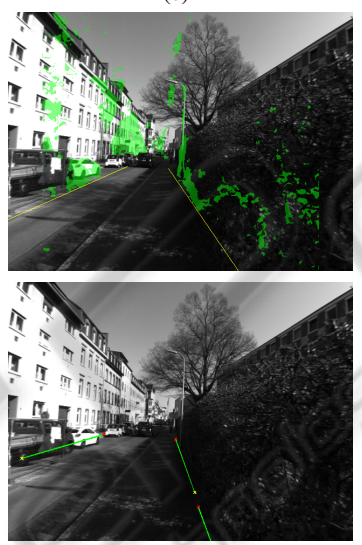

(g)

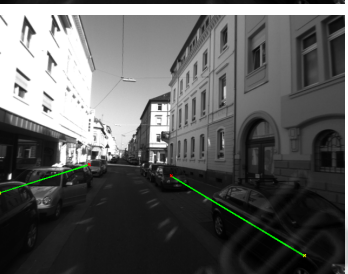

(d)
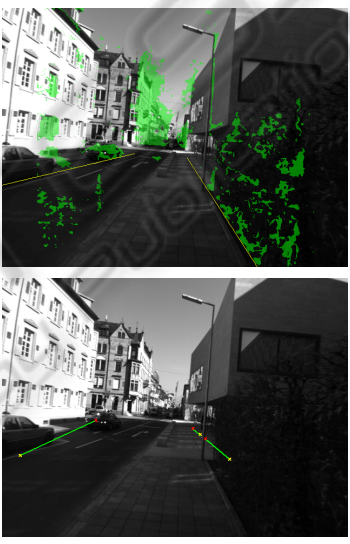

(h)

Figure 4: Results of RANSAC based planar segmentation (top row) and estimation of facade orientations using elevation maps (bottom row), which can be used in a subsequent step to generate an according surface.

\subsection{Iterative Least-squares}

The random nature of the RANSAC segmentation and the assumption of perfectly vertical buildings in case of the elevation maps prevent either approach to produce perfectly robust results. Nevertheless, both approaches normally yield an approximation of the main orientation of the buildings, which is accurate enough to optimize the estimated surface with a least squares estimator. To deal with the remaining outliers we optimize the plane hypotheses iteratively. While shrinking the plane to point distance threshold in every iteration, the optimization converges within a few iterations.

We verify the plane by comparing the normal angle deviation between the initial fit and the optimized fit. A false initial fit will lead to big deviations and can be rejected in this way.

\section{EXPERIMENTS}

In a set of experiments we compare the different approaches for plane estimation. Our experimental setup consists of a calibrated stereo rig with a short baseline of around $10 \mathrm{~cm}$ and a video resolution of 640x480px. To enlarge the field-of-view we deploy a wide-angle lens of $12 \mathrm{~mm}$ focal length. We obtain the dense disparity estimation using an off-the-shelf semi-global-matching approach (OpenCV).

\subsection{Evaluation}

We ran some tests on a dataset consisting of inner city scenes captured from ego-view to evaluate the applicability of the proposed approaches in some challenging scenarios. Processing video data from ego-view perspective especially has to be robust against occlusion and the high degree of clutter caused by parked cars or bikes, trees, or other dynamic traffic participants. Another issue in real life scenarios are the chal- 

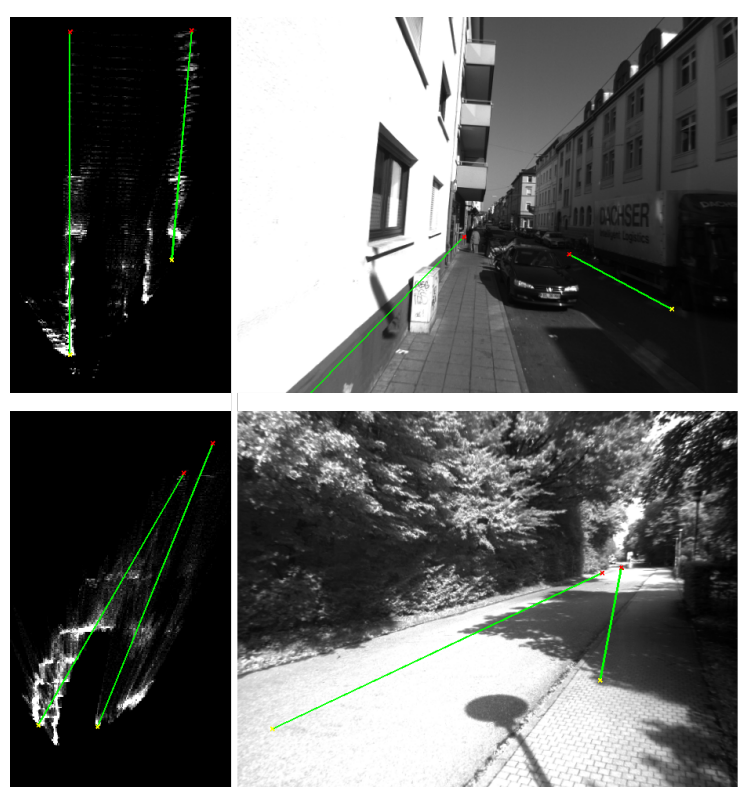

Figure 5: Left column: Elevation maps. Connected elements are found by Hough transformation and projected into the camera image (right column).

lenging light conditions, that often lead to over- and underexposed image parts in the same image. Figure 4 shows 8 scenes with the result of RANSAC segmentation in the top row, and the resulting facade orientation drawn from elevation maps in the bottom row.

\section{Planar RANSAC Segmentation}

The random plane selection in the RANSAC segmentation approach makes it difficult to draw quantitative conclusions about the performance. To underlay some numbers we picked five of the more difficult scenarios and evaluated the repeatability of the output. We ran the algorithm 50 times on each scenario and evaluated the number of correct wall estimations by manual supervision. We consider a wall as missed

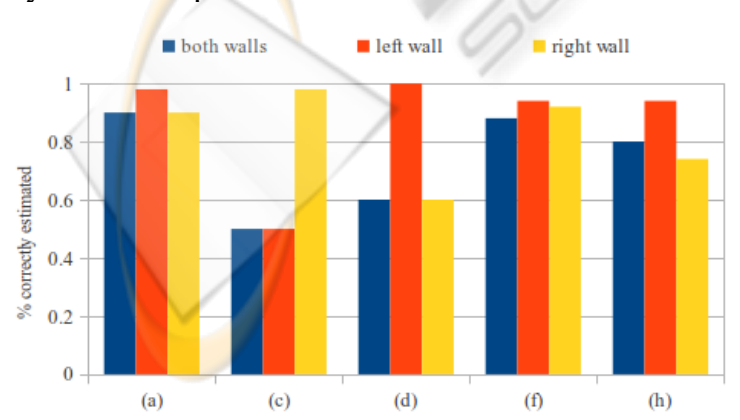

Figure 6: Evaluation of repeatability of planar RANSAC segmentation. Shown is the percentage of correctly determined walls in 50 repetitions, scenes correspond to Figure 4. when the estimated orientation deviates so strongly, that a subsequent optimization step will not converge close to the optimum.

Figure 6 shows the results for some scenarios taken from Figure 4. Scenario (c) is challenging in that the left building wall is hardly visible due to occlusion, and the visible part is overexposed. The right wall is found very robustly. The large amount of errors in detecting the right wall in scenario (d) can be explained by clutter, which often leads to planes fitted to the sides of the parked cars. Increasing the number of sampled planes per iteration would probably prevent this. The substantial gap on the right hand side in (h) explains the often missed right wall. In scenarios with mostly free view on the walls a rate of around $90 \%$ for both walls is realistic.

\section{Elevation Maps}

To rate the stability of wall estimation based on elevation maps we investigate two sequences of 200 and 500 frames, taken while travelling down a street canyon. In each frame we estimate the orientation of both walls, independent of the previous frame.

The first sequence consists of 200 frames and is mostly free of wall occlusions. The algorithm finds the correct wall orientation in all but 8 frames, that
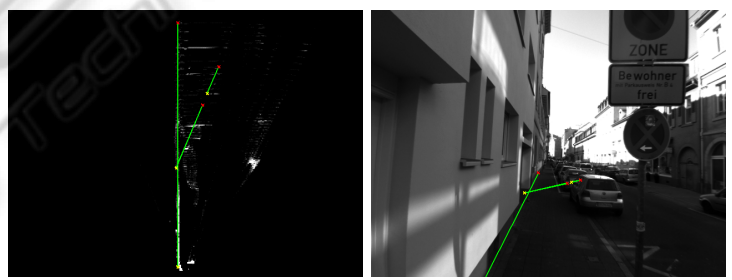

(a)

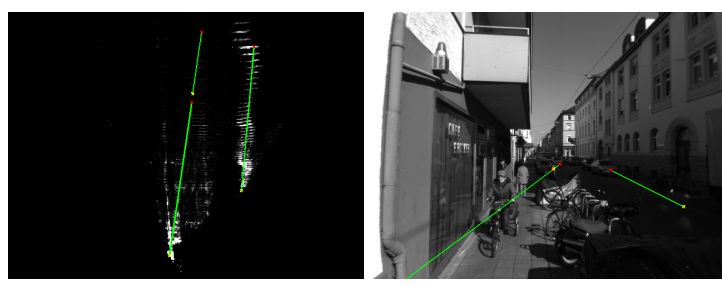

(b)
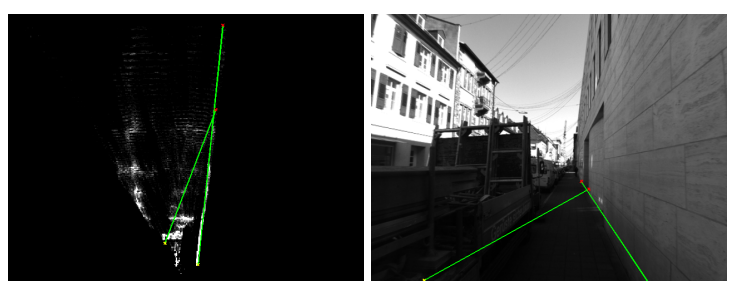

(c)

Figure 7: Failures in orientation estimation using elevation maps. 

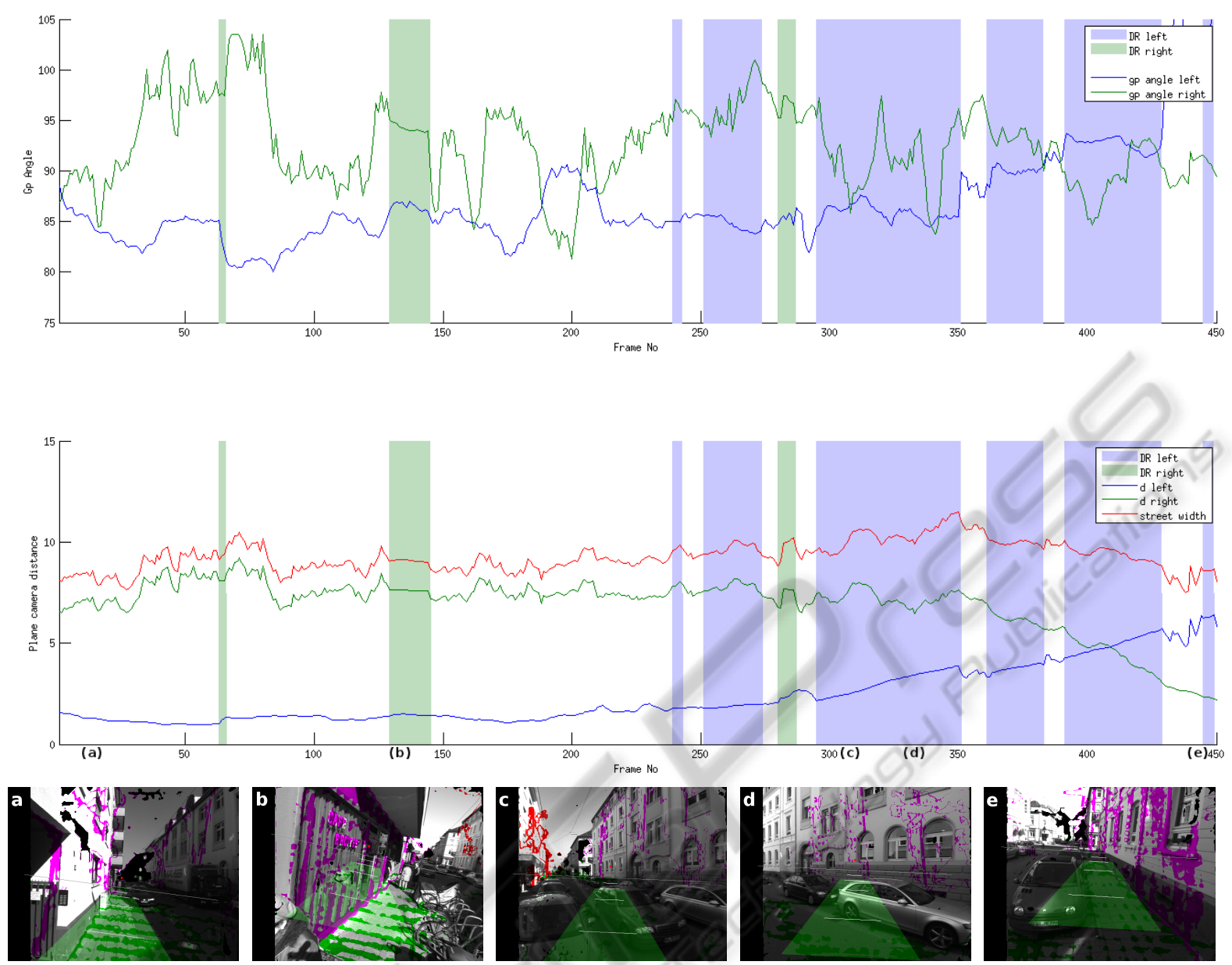

Figure 8: Plane parameters tracked over a sequence of 450 frames. The top diagram shows the groundplane angle, the bottom diagram the normal plane distance. Predicted parts due to walls being out of view are shaded.

were taken while passing a street sign (see Figure 7(a)). The second sequence consists of 500 frames and is more cluttered. The algorithm fails in around $10 \%$ of all frames to estimate one of the walls correctly. Reasons are always related to obstacles that were not filtered because they exceed the groundplane cut-off height, or obstacles that occlude the wall. See Figures 7(b) and 7(c) for two examples.

\subsection{Geometry Tracking}

The wall estimation is embedded as part of a realtime system, which also contains a module to estimate the camera motion between consecutive frames by running the visual odometry estimation taken from the LIBVISO2 library (Geiger et al., 2011b). Visual odometry provides the egomotion in all six degrees of freedom such that the camera poses of frame $n-1$ and $n$ are related via a translation vector $\vec{t}$ and a rotation matrix $R$.

Knowledge of the camera transformation allows to predict the current groundplane and wall parameters from the previous frame. The $X Y Z$-plane $\vec{p}_{t-1}=\{\vec{n}, d\}$, with surface normal $\vec{n}$ and distance $d$ from the camera origin, transforms into the current frame via

$$
\vec{p}_{t}=\left([R \mid \vec{t}]^{-1}\right)^{T} \vec{p}_{t-1}
$$

In a street canyon scenario we proceed as follows: We initialize the groundplane and planes for left and right wall with the methods described above. For the following frames we use the prediction as starting point for the iterative least squares optimization to compensate the inaccuracy of the egomotion estimation. To stabilize the process over time we store the best fitting support points in a plane history ring buffer and incorporate them with a small weighting factor into the subsequent least squares optimization. We reject the optimization when the plane normal angles of prediction and optimization deviate by more than $5^{\circ}$ in either direction, or the groundplane angle becomes smaller than $80^{\circ}$. Reasons for this to hap- 
pen are normally related to a limited view onto the wall, which either occurs when the wall is occluded by some close object (e.g. truck), or the cameras temporarily point away from the wall. If the optimization was rejected we carry over the prediction as current estimate and continue like that until the wall is in proper view again.

The estimated plane parameters for both walls in a sequence over 450 frames are plotted in Figure 8. The upper diagram shows the angle between groundplane and walls, the bottom diagram shows the plane distance parameters. The distances add up to the street width, for this sequence with a mean of $9.3 \mathrm{~m}$.

The sequence begins on the left sidewalk and ends on the right sidewalk after crossing the street. It contains several parts in which the walls are out of view due to the camera heading, some are shown in the screen shots. As explained earlier, these parts are bridged by predicting the parameters using the egomotion and are shaded in the diagram.

\section{CONCLUSIONS}

We have demonstrated two approaches towards estimating the local, geometric structure in the scenario of urban street canyons. We model the right and left building walls as planar surfaces and estimate the underlying plane parameters from 3D data points obtained from a passive stereo-camera system, which is replaceable by any kind of range sensor as long the uncertainties of reconstructed 3D points are known and can be considered.

The presented approaches are not intended as a standalone version. Their purpose is rather to separate a set of inlier points fitting the plane model to initialize optimization procedures as we applied in form of the iterative least-squares. By taking visual odometry in combination with a prediction and update step into the loop we are able to present a stable approach to keep track of groundplane and both walls.

Future work includes integrating the rich information offered by the depth-registered image intensity values and relaxing the assumptions implied by the street canyon scenario.

\section{ACKNOWLEDGEMENTS}

The work was supported by the German Federal Ministry of Education and Research within the project OIWOB. The authors would like to thank the "Karlsruhe School of Optics and Photonics" for supporting this work.

\section{REFERENCES}

Barinova, O., Lempitsky, V., Tretiak, E., and Kohli, P. (2010). Geometric image parsing in man-made environments. ECCV'10, pages 57-70, Berlin, Heidelberg. Springer-Verlag.

Borrmann, D., Elseberg, J., Lingemann, K., and Nüchter, A. (2011). The 3d hough transform for plane detection in point clouds: A review and a new accumulator design. 3D Res., 2(2):32:1-32:13.

Chumerin, N. and Van Hulle, M. M. (2008). Ground Plane Estimation Based on Dense Stereo Disparity. ICNNAI'08, pages 209-213, Minsk, Belarus.

Cornelis, N., Leibe, B., Cornelis, K., and Gool, L. V. (2008). 3d urban scene modeling integrating recognition and reconstruction. International Journal of Computer Vision, 78(2-3):121-141.

Fischler, M. A. and Bolles, R. C. (1981). Random sample consensus: a paradigm for model fitting with applications to image analysis and automated cartography. Commun. ACM, 24(6):381-395.

Geiger, A., Lauer, M., and Urtasun, R. (2011a). A generative model for $3 \mathrm{~d}$ urban scene understanding from movable platforms. In CVPR'11, Colorado Springs, USA.

Geiger, A., Ziegler, J., and Stiller, C. (2011b). Stereoscan: Dense $3 \mathrm{~d}$ reconstruction in real-time. In IEEE Intelligent Vehicles Symposium, Baden-Baden, Germany.

Gutmann, J.-S., Fukuchi, M., and Fujita, M. (2008). $3 \mathrm{~d}$ perception and environment map generation for humanoid robot navigation. I. J. Robotic Res., 27(10):1117-1134.

Hoiem, D., Efros, A. A., and Hebert, M. (2007). Recovering surface layout from an image. International Journal of Computer Vision, 75:151-172.

Hoover, A., Jean-baptiste, G., Jiang, X., Flynn, P. J., Bunke, H., Goldgof, D., Bowyer, K., Eggert, D., Fitzgibbon, A., and Fisher, R. (1996). An experimental comparison of range image segmentation algorithms.

Hough, P. (1962). Method and Means for Recognizing Complex Patterns. U.S. Patent 3.069.654.

Iocchi, L., Konolige, K., and Bajracharya, M. (2000). Visually realistic mapping of a planar environment with stereo. In ISER, volume 271, pages 521-532.

Labayrade, R., Aubert, D., and Tarel, J.-P. (2002). Real time obstacle detection in stereovision on non flat road geometry through "v-disparity" representation. In Intelligent Vehicle Symposium, 2002. IEEE, volume 2, pages $646-651$ vol.2.

Lee, D. C., Hebert, M., and Kanade, T. (2009). Geometric reasoning for single image structure recovery. In CVPR'09.

Murray, D. R. and Little, J. J. (2004). Environment modeling with stereo vision. IROS'04.

Poppinga, J., Vaskevicius, N., Birk, A., and Pathak, K. (2008). Fast plane detection and polygonalization in noisy $3 \mathrm{~d}$ range images. IROS'08.

Schindler, K. and Bischof, H. (2003). On robust regression in photogrammetric point clouds. In Michaelis, B. and Krell, G., editors, DAGM-Symposium, volume 2781 of Lecture Notes in Computer Science, pages 172-178. Springer.

Se, S. and Brady, M. (2002). Ground plane estimation, error analysis and applications. Robotics and Autonomous Systems, 39(2):59-71. 